\title{
Analysis of Online Texting among Bilingual Interlocutors
}

\author{
Rashad Ali Ahmed \\ English Department, Faculty of Education, Taiz University, Yemen \\ E-mail: rashadhse@ gmail.com
}

Abdu M. Talib Al-kadi

Language and Translation Center, Ibb University, Yemen

E-mail: findtalib@gmail.com

Received: September 6, 2016 Accepted: October 13, 2016 Published: October 17, 2016

doi:10.5296/ijele.v4i2.10167 URL: http://dx.doi.org/10.5296/ijele.v4i2.10167

\begin{abstract}
This study advances research on using language in online communication. It intended to analyze texting on WhatsApp in terms of linguistic and paralinguistic characteristics, the identity of interlocutors, and uses of emojis. Adopting a corpus-based approach to the study of language variation, data were collected from WhatsApp chat group of six male students enrolled in a Master's program at a graduate school in the Midwestern region of the United States. A total of 195 messages were garnered over a period of three months. Results showed laudable features of online texting which are deviant of the formal English orthographically, morphologically, syntactically, and phonologically. This supports previous claims that texting has adversely affected the structure of formal language. It was also found that emojis were used to complement words, impose additional meaning that mere words cannot convey or supplant written forms. Additionally, the way interlocutors used text messages reveals information about their identities. It also gives clues about their cultural, religious, and educational backgrounds.
\end{abstract}

Keywords: Emojis; interlocutors; linguistic features, texting; WhatsApp 


\section{Introduction}

Today, wired and wireless technology has provided a wide space for online communication. In fact, most of the world, under the auspices of the Internet now, has become a networked society where people contact and meet online more than they do face-to-face. Noticeably, many people today cannot bear to switch off their mobile phones; and if they do so, they will later be asked why! Such a tendency has redefined both private and social spaces and thus reshaped language. The fact that the Internet and its allied applications which surged globally have drastically changed the way people communicate and use the language, impacting both writing and speaking (Crystal, 2001, 2005, 2011, 2013; Waldrone, Kemp \& Wood, 2016). While some language researchers believe that the Internet has caused negative language change (e.g. Ali, 2012; Kern 2006; Prøysen, 2009), some others (e.g. Crystal, 2008) take the issue with the contention that it provides a platform which is conducive for massive contact, community building, and language change. However, online interactions lack facial expressions, gestures and conventions of body posture; these are the features of real time communication that make an integral part of delivering messages among interlocutors important when expressing ideas and opinions (Kern, 2014).

\section{Literature Review}

\subsection{Online Communication}

The Internet, the World Wide Web (abbreviated as WWW) and many wearable associated appliances have transformed face-to-face communication into patently electronic communication with unique linguistic and non-linguistic (paralinguistic) features, including the use of emoticons. Interlocutors tend to use various strategies to express themselves electronically. One of these strategies is the utilization of emojis. However, their semantic role is rather limited. For example, the basic smile can mean sympathy, delight, amusement and other types of emotion (Park, Fink, Barash \& Cha, 2013). Interlocutors use different semiotic devices which might be misunderstood. Not all of these devices are universal, though; this poses difficulties on cross-cultural communication to convey information smoothly across cyberspace to unknown recipients (Kavanagh, 2010). Put differently, unlike real communication, which is supported by what linguists call 'paralinguistic features' such as facial expressions, eye-contact, etc., virtual (online) communication lacks such communicational devices.

\subsection{Texting}

The pervasiveness of the Internet applications and tools has spawned disagreements among language researchers regarding its effects on the normal/usual usage of the language. Some believe that English varieties used on the Internet are a real threat to the standard varieties of English. Daily communication through Facebook, WhatsApp, Twitter, and $\mathrm{SMS}^{1}$, for instance, generally are less correct, less complex, and less coherent than other forms of

\footnotetext{
${ }^{1}$ SMS stand for short message service; in this study, however, it simply refers to short messages.
} 
language; this makes the written speech less structured, less formal, and more abbreviated (Ali 2012; Crystal, 2001; Herring, 2001; Kern, 2006; Prøysen, 2009). In a way, it has become fast and simple because the nature of these communication systems requires speed and simplification.

On the other hand, well established linguists such as the British David Crystal (2001, 2005) believe that this linguistic phenomenon occurring in daily English is a linguistic revolution. Although much of this 'electronic language' is touted as non-standard and highly deviant of the formal English, Crystal (2001) extoled its emergence; he has challenged some of previous authors' conclusions, arguing that this new discoursal use of language is prima facie evidence of the phenomenon that is likely changing the way we think about language in a fundamental way. To Crystal, the great change in English form and usage gave way to the emergence of a new branch of linguistics, Internet Linguistics (Crystal, 2011) which has created a host of terms associated with online communication.

Although online writing is widely believed to destroy the purity of English, Crystal (2005) surmised that "it should be exulting that the Internet is allowing us to once more explore the power of the written language in a creative way" (p.2). To illustrate, by chatting/texting, online communities have developed simple versions of words that are otherwise complex and cumbersome to type, such as the word [great] becomes [gr8]. Today's generation, according to Crystal (2005), are the ones who have "introduced several deviant spellings" (p.9) such as [kool $=\mathrm{cool}]$, [fone $=$ phone $]$, and $[\mathrm{B} 4=$ before $]$. Such typographic and weird spelling gave way to a common international language that Crystal initially called Netspeak, a variety of English used to communicate online. While Netspeak may be a bit dated in the twenty-first century realities, its rudiments are relevant to today's synchronous and asynchronous communication $^{2}$. Recently, the term 'textese' or 'texting' (Waldrone, Kemp \& Wood, 2016) have gained prominence over their antecedent 'Internetese' ${ }^{3}$ or 'Netspeak'. Crystal (2008, 2013) maintained that texting/textese is a newly born 'genre' of modern English and is not affecting the major part of the Standard (formal) English.

In 2009, Prøysen sketched out the impacts of texting on Standard English. The study reported that electronic interactions via emails, Internet chat and text messaging brought about the following noteworthy linguistic characteristics of 'textese':

- Use of abbreviations

- Incomplete sentences

- Contractions

- Nonstandard use of apostrophe

- The use of lower case letters in places of upper case letters

- Nonstandard spelling

- Exaggerated use of exclamation mark, period, and question mark

- Informal style of language

- The use of capital letters

\footnotetext{
Synchronous (real time) and asynchronous (deferred time).

3 A very informal style of English used on the Internet and used interchangeably with NetSpeak.
} 
Contradictorily with Crystal's (2008) positive viewpoint towards the invading 'textese', Prøysen envisaged that it erodes second language (L2) learners' ability to spell and punctuate correctly; and they also have difficulties in separating the language of text messaging from the Standard varieties of English.

\subsection{Linguistic Features}

The Internet English (Crystal, 2008, 2011) evoked the attention of several language researchers to examine it from different perspectives. Heidari and Alibabaee (2013) studied the linguistic and discoursal features of texting as used by Iranian EFL learners. The study analyzed 400 SMS with a focus on linguistic features like punctuation, truncation, initialization, logographic emotions, alphanumeric homophones, message complexity, and use of abbreviations. The results of the study showed that using new vocabulary and logographic emotions have become a leitmotif of the Iranian texters, especially females. The results unveiled discourse features which varied among male and female users. The study found that females used more complex sentences in their messages than male. Plus, the use of emotional signs and expressions were popular among the female participants. According to the authors, in the Iranian culture, "males are regarded as powerful, firm, strict and unemotional persons especially where they are in contact with other males, while females are regarded as kind, flexible, and emotional" (p. 68).

Similarly, Matu, Ong'onda, and Oloo (2011) studied some syntactic aspects of text messages in the Kenyan context. The study analyzed 160 messages collected from 40 Kenyan undergraduate students. Major findings of the study revealed that text messaging resulted in a paradigm shift in the traditional usage of language; new syntactic structures permeated into the linguistic continuum of Kenyan texters who exhibited (a) variations in the level of English morpho-syntactic (e.g. omission of pronouns and auxiliary verbs); (b) contractions as a way to condense language; (c) change of the word order; (d) usage of abbreviations in sentences; and (e) negligence of grammatical agreement between the lexemes used in the sentence structures that the participants created.

Tagg (2009) examined the language use in a corpus of text messages. The study examined 441 messages compiled from friends and family; the analysis went beyond the abbreviations and spelling variation. It elucidated other linguistic features which may define texting as a language variety. The study concluded that texters had recourse to craft text messages to fulfil speech-like purposes (use of speech-like grammatical features construes an appropriately informal arena), and, at other times, to fulfil transactional functions such as requests or coordinating future arrangements.

Gunraj, Drumm-Hewitt, Dashow, Upadhyay, and Klin (2016) explored the role of the period (.) in text messaging exchange (as a cue for pragmatic and social information). The data included a series of SMS exchanges collected from 126 Binghamton University undergraduates. The study found that text messages ending with a period (point) were perceived as being less sincere; the people who send periods (...) were thought of as heartless. This might be why interlocutors tend not to use punctuations sufficiently. 
While the studies of Matu, Ong'onda, and Oloo (2011) and Tagg (2009) are relevant to the topic the current study advocates, it is important to look into the phenomenon among Arabic-English bilinguals on the one hand, and scrutinize more paralinguistic features that the previous studies missed to highlight adequately.

\subsection{Paralinguistic Features}

Paralinguistic (i.e. non-semantic characteristics of the voice) and nonverbal (i.e. posture, gestures, and facial expressions) behaviors play important roles in conveying information about personality traits of the interlocutors. The paralinguistic devices (emoticons) have been observed in posts, tweets, comments, emails, and SMS. Derk, Bos, and Von Grumbkow (2008) analyzed how emoticons were used in online communication among friends and also when friends communicate with strangers. A total of 1251 participated in the study in the form of responses to an online chat. The findings revealed three obvious purposes of using such semiotic symbols:

a. Emphasizing a message.

b. Venting emotions.

c. Expressing humor.

The study concluded that the participants used emoticons as a way of compensating for the absence of facial expressions. According to this study, when friends chat with each other, they tend to use such signs more than chatting with strangers.

Likewise, Parkins (2012) observed six most common techniques of emotional expressions:

- Extensive use of punctuation markers (e.g.!!! and ?? ).

- Use of extensive full stops within a speech sample (e.g. .....).

- Use of capitalized words/phrases.

- Addition of the same letter within a word (e.g. yeeeeees).

- General use of emotions.

- Different means of expressing laughter (e.g. lol, hehe, haha)

Al Rashdi (2015) examined the forms and functions of emojis in SMS from the gender viewpoint. The data was taken from two groups based on gender: one male and one female; both groups were WhatsApp group members in Oman. The analysis showed that 121 emojis were used in both groups. Some of these emojis appeared only (or predominantly) in one group but not in the other, reflecting gender differences. Results of this study showed that women used emojis more than men. The study demonstrated that emojis serve not only as indicators of users' emotions but some other communicative functions. For example, they (emojis) indicate approval or disapproval of others' messages, responses to expressions of thanks and compliments, conversational openings and closings, and so on. The study also examined the forms and functions of repetition of emojis. These forms were found to serve as indicators of emotion intensity, enthusiasm, excitement, insistence, and solidarity. Besides, they were used to highlight a certain part of suprasegments. 


\subsection{Cultural Identity}

Not only have these additions enriched English language but also affected and are related to cultural identity. There are similarities between face-to-face (verbal) communication and the (non-verbal) conversational strategies in online interactions. These similarities are more pronounced in Japanese, for instance, which is testimony to how higher context cultures find these cues a necessity for smooth communication within a low context environment (Kavanagh, 2010). In contrast, the use of non-verbal contextual cues in low context cultures and as reflected in the data here was less relied on to promote and necessitate agreeable communication within this low context medium.

Park, Fink, Barash, and Cha (2013) investigated the semantic, cultural, and social aspects of emoticon usage on Twitter. The study included only emoticons expressing human facial cues in 15 eastern and western countries. Findings showed that facial expressions may not be universal but are used differently across various countries; also, they are not limited to conveying a specific emotion or used as jokes but rather are socio-cultural norms, whose meaning can vary depending on the identity of the speakers.

\subsection{Statement of the Research Problem}

Prior research has tackled some linguistic features of texting including clause combination, situational ellipsis, deixis, headers, and tails, vague language, response markers, fixed tags, and discourse markers (Carter \& McCarthy, 2006). Similarly, a common paralinguistic feature some previous studies addressed is the use of emojis (Al Rashdi, 2015; Derk, Bos \& Grumbkow, 2008). Although the study of linguistic characteristics of texting and emojis is no novelty, previous studies have only scratched the surface of the topic. This study advances research within the dimension of linguistic and non-linguistic features of WhatsApp-based texting which has become the linchpin of today's personal communication. The study delved into the uses of texting in terms of linguistic characteristics, the identity of the interlocutors, and the uses of emojis. It investigated why interlocutors tend to use emojis and how they make up for the absence of paralinguistic features of real time communication.

\subsection{Research Questions}

The study addressed the following questions:

1. What are the linguistic characteristics of text messaging among bilinguals?

2. What are the commonly used emojis in text messages among bilinguals? How are they used?

3. How do interlocutors uncover their identity while texting?

\section{Method}

This study, through a corpus-based approach to the study of language variation, examined the linguistic as well as paralinguistic characteristics of WhatsApp-based text messaging as one 
of the Web 0.2 applications. Specifically, it elucidated how the sentence structure of SMS is different from that of formal English writing.

\subsection{Participants}

Six male students doing their Master's at a graduate school in the Midwestern region of the United States participated in the study. They created a WhatsApp group for a class that they were all registered in. The purpose of this group was to discuss issues related to a particular class, namely historical linguistics course) but they ended up using it to socialize with each other and discuss matters related to life in general. The interlocutors were bilinguals; five of them were Arabic-English users, and one speaks Spanish and English with few words/phrases in Arabic. Being a member of this group, one of the two authors ${ }^{4}$ asked the other group members for their consent to use their texts for research purposes. Having obtained consent, the authors gathered the data and performed a qualitative analysis.

\subsection{Material (Corpus)}

A total of 195 messages were collected over a period of three months. During this period, the participants actively used the group in question. The data included several settings; the participants talked (online) about several topics. The data were rich with emoticons which is a unique characteristic of texting.

\section{Results and Discussion}

The study is a detailed investigation of short messaging on WhatsApp. Guided by the design of the study, descriptive statistical analysis was used to calculate the frequencies and percentages of the occurrences of the phenomenon in the data collected. To protect the identity of the interlocutors, only the initials of their first names were revealed. In the analysis, the statistical figures were incorporated in a qualitative analysis with a flashback on the literature and from the part of the authors.

\subsection{Q \#1: What are the linguistic characteristics of text messaging among bilinguals?}

This question sought to determine the distinguishing features of text messaging. A major one, this study reveals, is shortness of sentences. The sentence structure of SMS is short and lacks the basic rules of formal writing. The data showed that none of the participants followed the formal English grammar. In terms of sentence structure, an interlocutor may write an affirmative sentence and put a question mark at the end to show that he is asking a question and not making a statement. At the beginning of the conversation (see Figure 1), R asked sarcastically, "Anybody home???"

\footnotetext{
${ }^{4} \mathrm{He}$ is currently living and doing his Ph.D. in the U.S.
} 


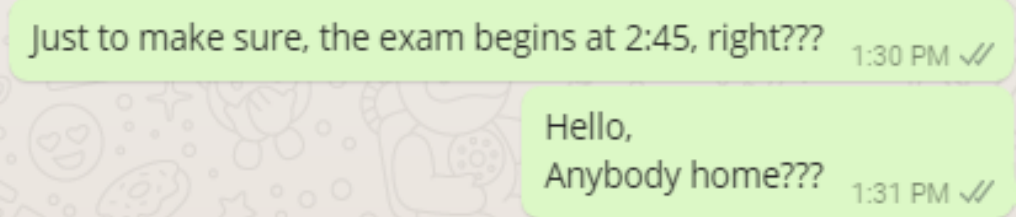

Figure 1. R's First Message to the Group

Another characteristic of texting is lack of deviation of punctuations. Most of the interlocutors do not use punctuation marks, or may use them defiantly of the conventional rules. In messages 13 and 14 (see Figure 2), the interlocutor asked a question in the first sentence and did not use a question mark. Similarly, they made a statement in the second sentence and did not use a period. Based on the corpus, the participants seem to have used questions and exclamation marks more than any other types of punctuations. Even though some interlocutors still use few punctuation marks, the general trend shows that texting is more about conveying messages and not about grammar.

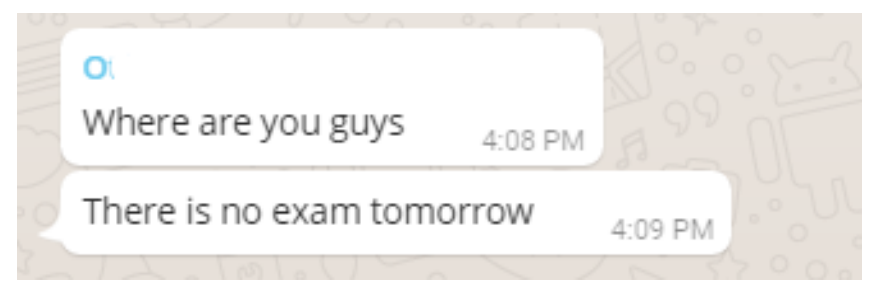

Figure 2. Lack of Punctuation on online conversation

Texting is also known for informality of opening or closing a conversation. To compensate the absence of norms of formality in SMS, punctuations seem to fulfil this shortcoming. For instance, as indicated in the illustration at hand, the question marks likely attract the attention of online participants (interlocutors) at the time of sending the message. This example shows that the interlocutor is establishing a context as if he was entering a house and asking if anyone was available there. This indicates that there is a tendency to liken online communities to real life situations.

Examining these SMS at the levels of pronunciation and word structure yield some findings. Morphologically, the use of shortened forms was common among the participants. In one message (see Figure 6), the speaker used $\mathrm{Jk}$ to state that he was "just kidding" about his previous statement, i.e. they used acronym (the initials $\mathrm{J}$ and $\mathrm{K}$ ). In another example (see Figures 3), J used 'hist ling.' to refer to 'historical linguistics', and used ' $r$ ' to refer to the pronoun 'are'. Other examples included the following table. 


\section{Macrothink}

Table 1. Examples of abbreviated forms in WhataApp texting

\begin{tabular}{|l|l|}
\hline \multicolumn{1}{|c|}{ Shortened forms } & \multicolumn{1}{c|}{ Examples from the study corpus } \\
\hline gonna' "going to" & $\begin{array}{l}\text { O: J said he would be late because he gonna finish } \\
\text { reading historical linguistics. }\end{array}$ \\
\hline ur' "your" & : I agree with ur suggestion \\
\hline plz' "please" & J: A, plz can u give a ride? \\
\hline
\end{tabular}

Phonologically, all these examples show that interlocutors tend not only to use more shortened forms but less attention to pronunciation marks. For example, they tend to use 'plz' instead of 'please' because please is pronounced with a $\mathrm{z}$ sound.

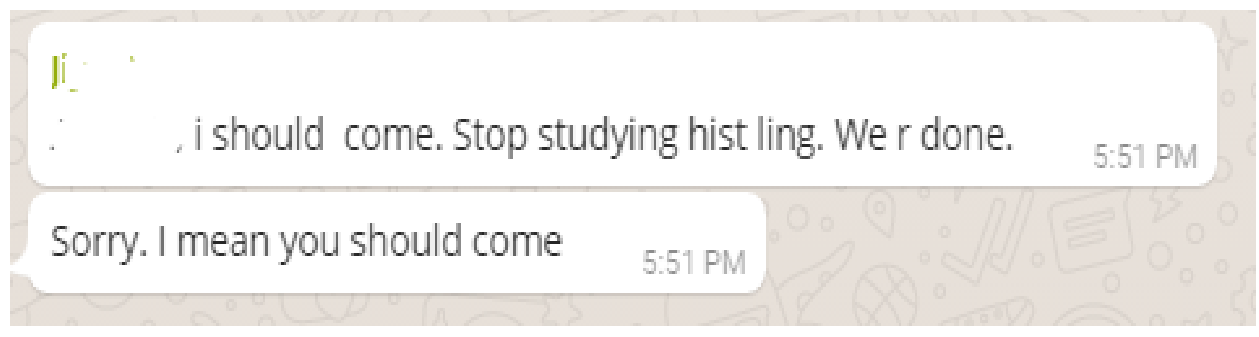

Figure 3. An Example Using Shortened Forms on WhatsApp

These morphological and phonological characteristics (shortening, acronyms, and clippings) were investigated in prior research studies (e.g. Prøysen, 2009; Crystal, 2005). The examples above show that the uses of text messages differ from formal writing in a way that most interlocutors do not follow the syntactic, morphological and phonological norms of formal English. Most interlocutors tend to use abbreviated forms and pay less attention to the usual usage of punctuation marks. The data of the current study contradicts with Parkins' (2012) findings that speakers use punctuation marks extensively; no one of the interlocutors in the current study paid enough attention to punctuation. Nevertheless, differences between sentence punctuation, perhaps, have something to do with emotion; when texting, speakers are not usually judged on how well they use grammar or punctuation but on how they use shortened forms and emojis.

Scrutinizing the corpus shows that texting resulted in a little fraction of the language that has been changed due to texting. Crystal (2013) asserted that even though texting influenced language in many ways; it has not changed English but added to the multiport of the language, making it richer and bigger. In other words, the vast majority of the language words, structures and other language aspects did not change as a result of texting. Texting is obviously influenced by the fast mode of modern hasty life situations.

4.2 Question 2: What are the commonly used emojis in text messages among bilinguals? How are they used? 
With respect to the second research question, it was found that WhatsApp texting employ several emojis to fulfil some purposes. Table 2 outlines all the emojis used in the corpus and the frequencies of those emojis, i.e. 'how many times' the interlocutors used them while texting (insofar as the corpus in focus is concerned).

Table 2. Common Emojis in the Corpus

\begin{tabular}{|c|c|c|}
\hline & Emoji & Frequency of use \\
\hline 1 & $\mathrm{cb}$ & 4 times \\
\hline & $\mathrm{db} \mathrm{db}$ & 1 time \\
\hline 3 & de de de de de & 1 time \\
\hline 4 & ()ㅜㅇ & 2 times \\
\hline 5 & (부요웅 & 1 time \\
\hline 6 & $\theta \theta$ & 1 time \\
\hline 7 & (앙 & 2 times \\
\hline 8 & $\therefore$ & 1 time \\
\hline 9 & 상 & 1 time \\
\hline 10 & ac) & 1 time \\
\hline 11 & $\Leftrightarrow$ & 1 time \\
\hline 12 & 9 & 1 time \\
\hline 13 & & 1 time \\
\hline 14 & 8 & 1 time \\
\hline 15 & 32 & 1 time \\
\hline 16 & 6 & 1 time \\
\hline
\end{tabular}

As data in the table shows, 12 different types of emojis were used, with thumbs up as the most frequently used (It was used 6 six times followed by smiley face, and heart emoji). In four of the contexts where it was used, it indicated approval. For example, the message shown in Figure 4 illustrate that 'thumbs up' $C$ can be used as a sign of agreement or approval of something; ' $A$ ' agreed to a proposal made by $\mathrm{O}$ to meet at Red Lobster from 6 7. A followed this emoji by writing "I agree with ur suggestion," which intensifies the meaning of emojis.

In another context, the emoji of 'thumbs up' carried the meaning of "good job." For example, in one of the messages, the interlocutor used 5 thumbs ups as a reply to a congratulation made by a previous message. This was as if the interlocutor was saying "good job everyone." This was confirmed by the interlocutor's following statement "congrats everyone!!" This seems to be consistent with Al Rashdi (2015) findings that the repetition of the same emoji in one message indicates intensity of emotions. The examples above show that the speakers intend 


\section{Ml Macrothink}

International Journal of English Language Education

ISSN 2325-0887

2016, Vol. 4, No. 2

to use emojis along with written texts to confirm the meaning of their messages. The question is: aren't emojis enough to convey the message? Apparently, the interlocutors may think that it is insufficient to send an emoji; and that is why they follow it with a written text, or it might mean that the interlocutors are trying to emphasize their message by using both emojis and written text.

The second commonly used emoji was the smiley face $\theta$. It was used 5 times in the corpus collected. In one context, it was used along with a grinning face. A smiley face usually shows that the person is happy with the situation, or the situation is humorous. It can also be used when the person is being sarcastic. For example, in one setting, the interlocutors were sarcastically speaking to each other on who was smarter than the other; they would usually use a smiley face after each sarcastic statement.

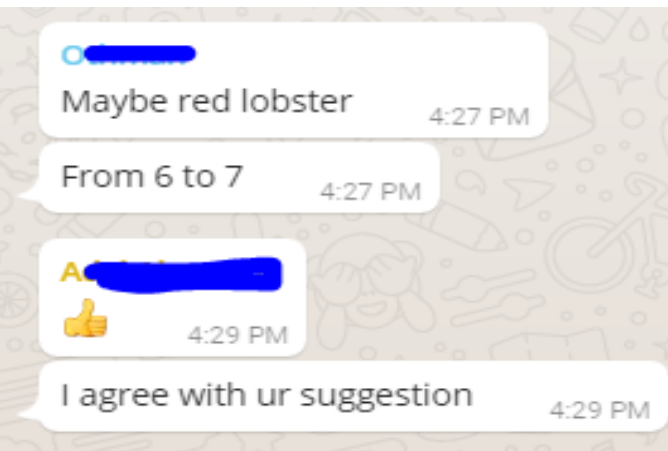

Figure 4. Emojis Showing Gratitude

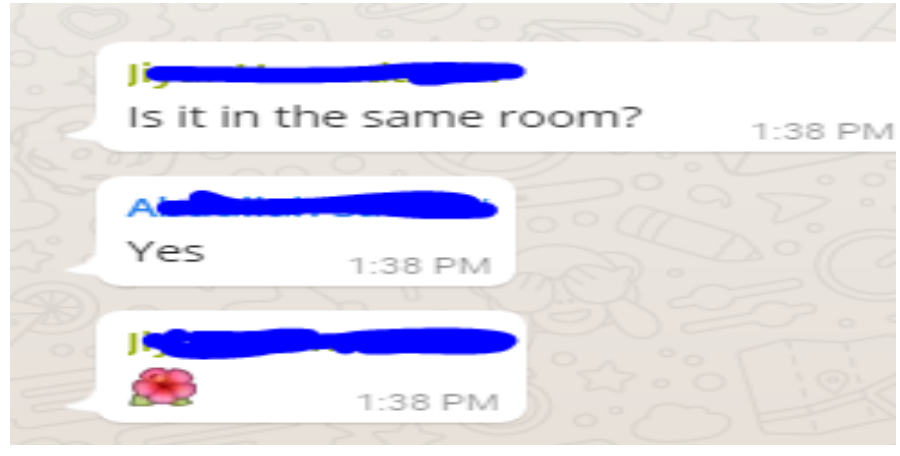

Figure 5. An Example Showing Emojis without Texts

While Figure 4 illustrates using emojis along with SMS, emojis can be, in some cases, used without text and they still carry meaning. In the messages shown in Figure 5, J asked a yes/no question about where they were going to meet, A answered with a yes, and then $\mathrm{J}$ replied with a flower without any other text . So, the flower meant "thank you." In some cultures, people might not perceive receiving a flower as a 'thank you' message; rather, it might mean something totally different. This leads to some assumptions about the cultural aspects text messages can reveal about the interlocutors.

The examples given above show that emojis are not only used for expressing emotions, but also have other communicative functions. For instance, the use of thumbs up can indicate an approval of a previous proposal or can be used as a way of praising someone as if the person is saying "good job". Emojis can also be used to show gratitude, excitements, sarcasm, celebrations, etc. These findings are in accord with the results of Al Rashdi (2015) indicating that emojis express not only emotions but they have some other communicative functions, such as showing intensity of emotions, expressing enthusiasm and excitement, adding emphasis, etc. In a nutshell, emojis were commonly used to (a) complement a phrase/word/expression; (b) impose additional meaning that mere words cannot convey; (c) supplant written forms.

\subsection{Q3: How do interlocutors uncover their identity while texting?}




\section{Mll Macrothink}

On the question of identity, evidence discerned from the corpus shows that the interlocutors expose their identities through text messaging in several ways. Based on the data collected, the participants' identities can be seen through their uses of sarcasm, L1, and speech acts. The use of sarcasm was apparent in A's messages to J (see Figure 6). They all seemed to have finished an exam but did not like the class. So, A started the conversation by saying that he was still reading more about the class but followed that with Jk (just kidding); J burst into laughter; he also replied with a rhetorical question. The conversation continued with A, sarcastically, saying that the professor made him love historical linguistics; and J replied with Hahahahah. This indicates that A was unhappy with that class and so was J. This example can also reveal some information about the identity of the speakers as being sarcastic, funny, etc.

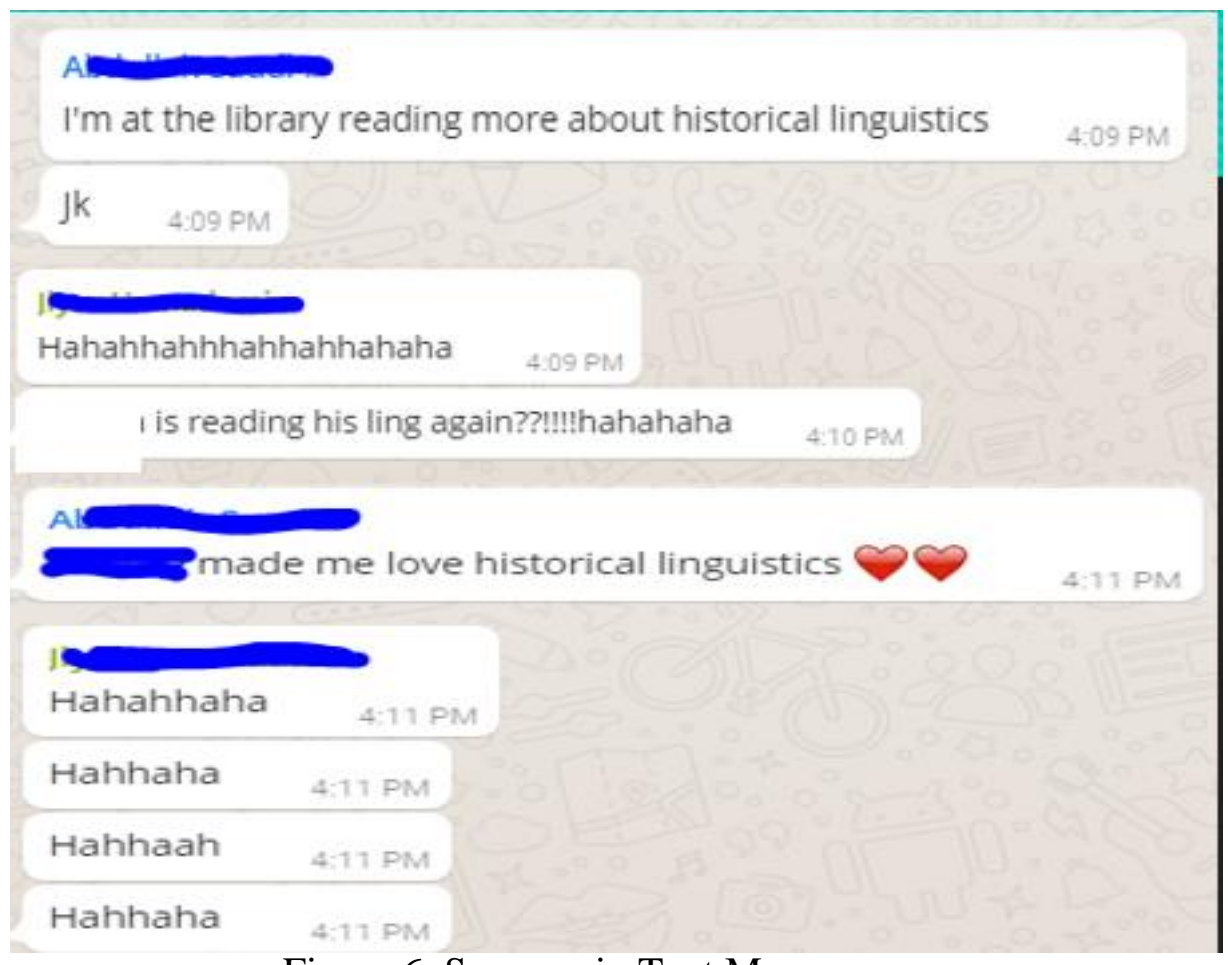

Figure 6. Sarcasm in Text Messages

Similarly, texting can uncover the ideological background of texters. After being invited for dinner by other interlocutors, A replied with "Assalam alikom" (Arabic phrase) an apology for not replying quickly, a wish, and then a reason why he might not be able to join them for dinner (see Figure 7). "Assalam alikom" is a greeting in A's first language but it was written in Roman alphabet. This specific 'greeting' tells something about A's identity. The greeting is in Arabic language which literally means "peace be upon you." It is considered to be the main religious greeting of Muslims all over the world. A possible explanation for this might be that the interlocutor wanted to show his identity and his religious values in accepting or refusing the invitation. After greeting, he apologized for refusing the invitation; and he gave a reason for replying late. As can be observed, the speaker indirectly and politely refused the invitation (implying) by saying, "you guys have fun and enjoy dinner. I'll be busy until 9 at least." In Arabic culture, it is considered rude to refuse an invitation unless one has a good reason to do so, especially if it were an invitation by a close friend or a relative. This is what 
actually happened in this conversation where A tried to be so polite in his reply so that it would not be considered rude. The speaker used a heart emoj $\vartheta$ after he wished they would have fun and enjoy dinner. It shows sincere wishes. The use of a heart emoji in some cultures may only be used to indicate romantic relationships, but in other cultures (e.g. Arabic) it may have more functions such as showing affection, respect, sincerity, etc. So, the use of emojis can provide some clues about the cultural differences and identities of the interlocutors.

The other person replied with "Have fun. We will meet another time inshallah." The use of "Inshallah" here was a kind of accommodation to A; "inshallah" is an Arabic phrase which means "God willing" This religious phrase is very common; it is being used not only by Arabic speakers but also Muslims around the world. It shows the religious identity of the person using it. It is a discourse marker that can be used in different contexts. In this context, it was used as a wish that God may bring them together one day. In other contexts, 'inshallah'

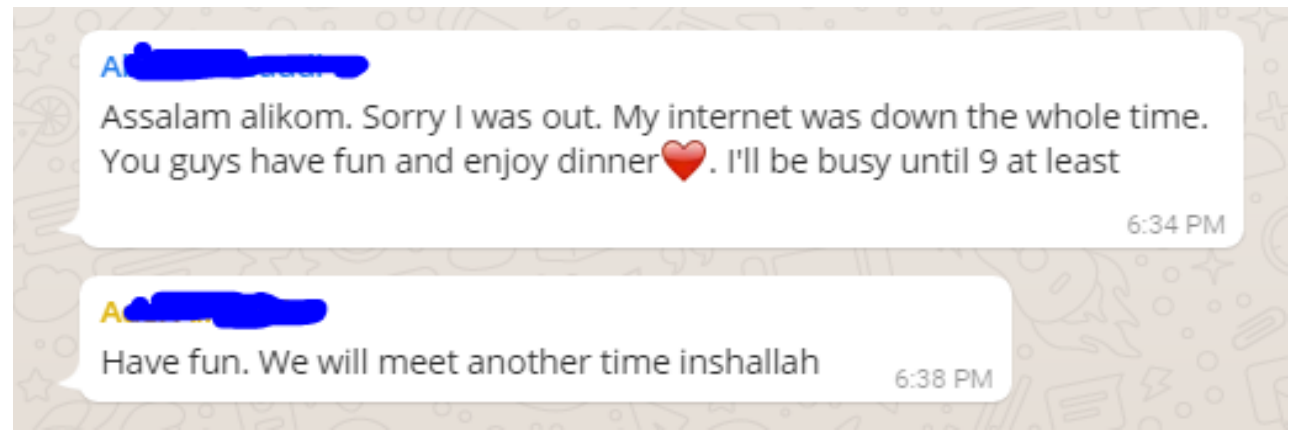

Figure 7. An Example Showing L1 and Speech Act in Text Messaging

can also mean that the speaker was not sure of doing something and refers it to God to help in making decisions. In other words, it reflects the interlocutor's faith in all what he does. It can also mean that the speaker is not willing to do something unless it gets changed by God. So, the use of this discourse marker depends on the context and it shows the religious identity of the person using it. It was not used in English because using religious texts in their original language always has authentic meanings; it is perceived stronger than if it were translated to another language.

In the conversation shown in Figure 8, the interlocutor started a conversation using L1 (the only Spanish participant in the group in question). He uttered the Spanish "Cómo estan", which means, 'how are you' in English. This interlocutor knew that all of the other participants do not speak Spanish but he seemed to have used it as a way of showing his identity as a Spanish speaker. He apparently knows little Arabic as he followed his Spanish greeting with an Arabic translation written in Roman alphabet "Kef halek." Interestingly, Kef halek is used for addressing a female in Arabic while in fact all the participants in the group were males. Since the group members know that is unfamiliar with the 'muscularity' of this phrase not knows much about Arabic, no one was offended. So, besides showing his identity as a Spanish speaker, $\mathrm{O}$ wanted also to show his respect for the Arabic speakers in the group by using their language. The next speaker seemed to have accommodated $\mathrm{O}$ by using Spanish 


\section{Ml Macrothink}

language; he seemed to not know Spanish since he used English and Spanish and he misspelled the second phrase. They all then switched to using English (as a common language of contact).

An interesting finding that deserves more highlight is that the interlocutors start conversations with something that relates to their cultural, religious and educational identity. In this example, the speaker wanted to expose himself as a Spanish speaker. Likewise, in Figure 7, the speaker showed himself as a person who not only speaks Arabic but also is a Muslim.

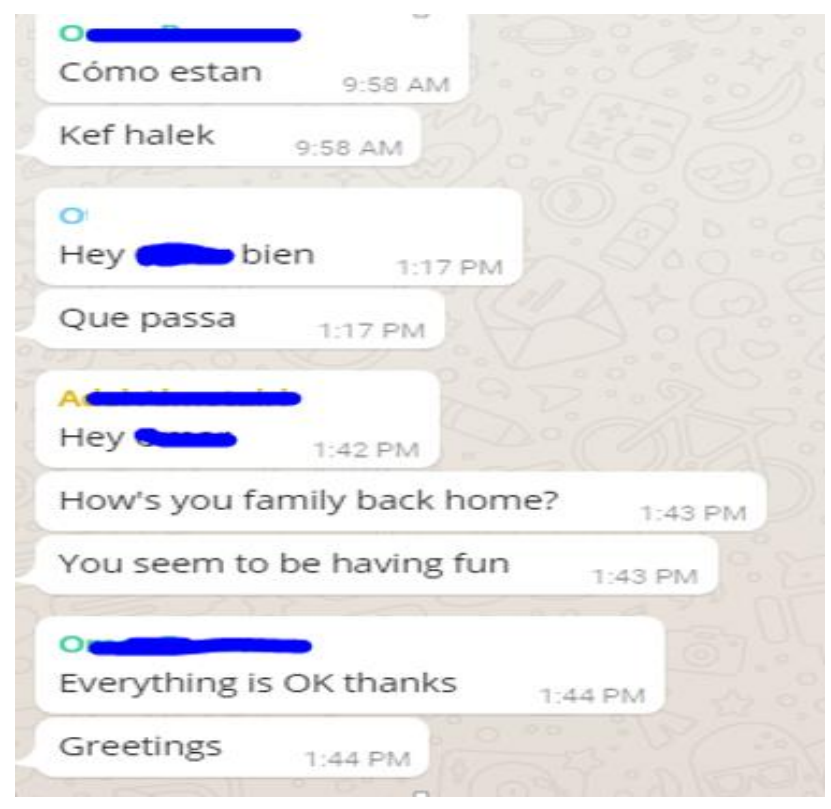

Figure 8. An Example Showing Code-switching in Text Messages

Figures 7 and 8 show how text messages can reveal some indicators about the identities of the interlocutors: their cultural, educational, and religious backgrounds which play significant roles in how they text each other. Also, the meaning of emojis is constructed through the interlocutors' culture and their use of the first language (L1).

\section{Conclusion}

This study demonstrated the utilization of text messaging in terms of linguistic and paralinguistic characteristics, identities of interlocutors, and the use of emojis. Although the study is based on a small sample of participants, the findings revealed laudable features that distinguish SMS texting from formal/standard varieties of English, such as using shortened forms of language which are orthographically, morphologically, syntactically, and phonologically deviant of the formal discourse. In texting, interlocutors pay less attention to punctuations and grammatical rules; they are likely more careful to convey their messages in a way that resembles face-to-face conversations. They do so by using paralinguistic features, primarily emojis.

The study has gone some way towards enhancing understanding of the linguistic and paralinguistic features of texting. It confirms previous findings and contributes additional 
evidence suggesting that texting is a linguistic phenomenon of unique characteristics. It supports the argument that texting is a new genre of English; it includes features such as emojis and excessive punctuations that are part of speech and not formal English writing. It also backs up prior claims that texting does not constitute a negative influence on the formal language; rather, it signals a rise of new language that is being used excessively by the internet-oriented generation (Crystal, 2008; 2013). Similarly, this paper makes noteworthy contributions to research on texting in terms of using emojis; it was found that emojis were used not only for expressing emotions, but also for other communicative purposes. Emojis can also be used to show gratitude, excitement, sarcasm, celebrations, etc. The study should also prove to be particularly valuable to sociolinguistic studies; the way interlocutors use text messages reveals some information about their different identities. People from different cultures perceive and employ facial expressions in unique ways. The data show that the interlocutors' uses of code-switching, speech acts, and sarcasm give clues about the cultural, religious, and educational identities of the speakers.

Although the study, theoretically, achieved its stated objectives, it has provided opportunities to plunge headfirst into some other related issues the study failed to address thoroughly. The limitations accruing from the sample size and data collection, or even, the characteristics of the participants, provide room for further research. The study's sample included 6 participants and 195 messages and participants were all males. A more comprehensive study would include a bigger sample that consists of equal number, maybe more, of males and females from different cultures which might enrich this topic and yield more interesting results.

\section{References}

Ali, J. K. (2012). Influence of information and communication technology (ICT) on English language structure (PhD dissertation). Aligarh Muslim University, Aligarh, India.

Al Rashdi, F. (2015). Forms and functions of emojis in WhatsApp interaction among Omanis. $\mathrm{PhD}$ dissertation. Retrieved from ProQuest Dissertations and Theses database (Document ID 1703437877).

Carter, R. M. McCarthy (2006). Cambridge grammar of English: A comprehensive guide. Cambridge: CUP.

Crystal, D. (2001). Language and the Internet ( $1^{\text {st }}$ ed.). Cambridge: CUP.

Crystal, D. (2005). The scope of the internet linguistics. Paper presented at the American Association for the Advancement of science meeting.

Crystal, D. (2008). Texting. ELT Journal, 62(1), 77-83. http://dx.doi.org/10.1093/elt/ccm080

Crystal, D. (2008). Txtng: The gr8 db8. Oxford, UK: Oxford University Press.

Crystal, D. (2011). Internet Linguistics: A student guide. New York, NY: Routledge. 
Crystal, D. (2013, November 9). The effect of new technologies on English (from the interview with David Crystal in Belgrade). Available on YouTube https://www.youtube.com/watch?v=qVqcoB798Is

Derk, D., Bos, A. R., \& von Grumbkow, J. (2008). Emoticons in computer-mediated communication: Social motives and social context. Cyberpsychology \& Behavior, 11(1), 99-101. http://dx.doi.org/10.1089/cpb.2007.9926

Gunraj, D, Drumm-Hewitt, A., Dashow, E., Upadhyay, S., \& Klin, C. (2016). Texting insincerely: The role of the period in text messaging. Computers in Human Behavior, 55, 1067-1075. http://dx.doi.org/10.1016/j.chb.2015.11.003

Heidari, M., \& Alibabaee, A. (2013). Linguistic and discoursal features of text message language created by Iranian male and female SMS users. Sheikhbahaee EFL Journal, 2(1). http://shbu.ac.ir/efl/efl3/4.pdf

Herring, S. C. (2001). Computer-mediated discourse. In D. Schiffrin, D. Tannen, \& H. E. Hamilton (Eds.), The handbook of discourse analysis (pp. 612-634). Malden, MA: Blackwell.

Kavanagh, B. (2010). A Cross-cultural analysis of Japanese and English non-verbal online communication: The use of emoticons in weblogs. Intercultural Communication Studies, 19(3), 65-80. http://web.uri.edu/iaics/files/05barryKavanagh.pdf

Kern, R. (2006). Perspectives on technology in learning and teaching languages. TESOL Quarterly, 40(1), 183-210. http://dx.doi.org/10.2307/40264516

Kern, R. (2014). Technology as pharmakon: The promise and perils of the Internet for foreign language education. The Modern Language Journal, 98(1), 340-357. http://dx.doi.org/10.1111/j.1540-4781.2014.12065.x

Matu, P. M., Ong'Onda, N. A., \& Oloo, P. A. (2011). Syntactic aspects in text messaging. World Journal of English Language, 1(1). http://dx.doi.org/10.5430/wjel.v1n1p2

Park, J. Fink, C., Barash, V. \& Cha, M. (2013). Emoticon style: Interpreting differences in emoticons across cultures. Proceedings of the Seventh International AAAI Conference on Weblogs and Social Media., 466- 475.

Prøysen, S. (2009). The impact of text messaging on Standard English ( $\mathrm{PhD}$ dissertation). Department of foreign languages, University of Bergen, Norway.

Tagg, C. (2009). A corpus linguistics study of SMS text messaging (Phd dissertation), School of English, Drama, and American and Canadian Studies, the University of Birmingham.

Waldrone, S., Kemp, N., \& Wood, C. (2016). Texting and language learning. In, A. Georgakopoulou \& T. Spilioti (Eds.), The Routledge handbook of language and digital communication (pp. 180-194). New York, NY: Routledge. 


\section{Copyright Disclaimer}

Copyright for this article is retained by the author(s), with first publication rights granted to the journal.

This is an open-access article distributed under the terms and conditions of the Creative Commons Attribution license (http://creativecommons.org/licenses/by/3.0/). 